\title{
Controlled Entropy and Gauge Theory in Recycling Condensed Matter by Solar Energy
}

\author{
Nilo Sylvio Costa Serpa ${ }^{1,2,3}$ \\ 1. GAUGE-F Scientific Researches, Brazil \\ 2. Institute of Science and Technology, Universidade Paulista, Brazil \\ 3. Faculdades ICESP, Brazil
}

\begin{abstract}
This article discusses the physical principles to be considered in a thermodynamic engineering solar system running under operational control algorithms designed to minimize entropy rates. The proposed plant is based on scientific foundations established by thermodynamics and classical field theory, and empirical disciplines that deal with properties of chemical elements and with technologies of calorific capture from remote sources. The gauge principle was applied in theoretical formalization as an essential tool in the control background of the entropy rates involved. The industrial system presented was planned to recycle condensed matter within the scope of a solid waste management policy.
\end{abstract}

Key words: Waste, thermodynamics, gauge principle, pyrolysis, solar energy, cleaner production.

\section{Nomenclature}

$\begin{array}{ll}\mathcal{L}: & \text { Lagrangian density } \\ \mathcal{D}: & \text { Covariant derivative } \\ \mathcal{S}: & \text { Action } \\ \mathrm{S}: & \text { Entropy } \\ \mathcal{A}_{q}: & \text { Gauge field } \\ \mathfrak{F}: & \text { Field strength (kinetic term) }\end{array}$

\section{Greek letters}

$\begin{array}{ll}\xi: & \text { Caloric field } \\ \varphi: & \text { Irreversibility coefficient }\end{array}$

\section{Introduction}

The implementation of the so-called SWM (solid waste management) is recognized as being of extreme importance for the preservation of public health and quality of life. The SWM requires skilled professionals, consisting of a set of management procedures (including a subset of medical waste management procedures, or, abbreviated, "MWMP”), based on

Corresponding author: Nilo Sylvio Costa Serpa, Ph.D., professor, research fields: quantum gravity, quantum computing, cosmology and thermal systems engineering. scientific, technical, regulatory and legal principles to minimize the production of waste and the risks involved in its disposal, efficiently protecting workers and preserving public health, natural resources and the environment in general, at low cost. In large cities of underdeveloped countries, where garbage became an increasingly critical issue, dumpsites and riverbeds remain the more frequent destinations of waste, endangering the health of coastal people and thousands of human beings who live at the expense of urban waste.

Several authors have been intensively addressing the problem of solid waste management since the 1990s [1-6]. Especially with regard to nosocomial sweepings, many attempts have been made to perform locally medical waste treatment. Cement works incinerators and blast furnaces used in steel industry have been suggested as local solutions [7], but failed in terms of safety in the process of waste loading and in the treatment of emissions. In particular, conventional incineration leads to the environment numerous substances, many of which, if not treated properly, stay with high toxic potential [8]. There are pollutants 
particularly persistent, that is, with molecules that accumulate in the food chain such as dioxins, furans and coplanar polychlorinated biphenyls [7]. The only effective form to optimize the process to reduce the formation of these substances is to guarantee eradication temperatures far above $800{ }^{\circ} \mathrm{C}$, preventing the formation of effluent gases at temperatures of 200-400 ${ }^{\circ} \mathrm{C}$ that occurs in combustion. Only by atomic disruption we could control the ultimate condition of such materials, recombining them to reduce global entropy rate.

This article aims to show from which theoretical principles it can be made a maximum control of entropy in an industrial process of condensed matter recycling, showing briefly the project of a plant for solid waste processing.

\section{The Gauge in Thermodynamics and the Prototype of the Waste Recycling System}

After the fleeting euphoria and the naive enthusiasm of the "atoms for peace" idea, typical of the late 1950s, the intricate techno-political reality of atomic energy became obvious to most people, leading humanity to seek cleaner energy technologies less affected by military interests. Thus, the use of the so-called "renewable sources" has become imperative for most nations, not only because of the increasing need for energy, but also because of the need for means of obtaining energy that are less aggressive to the environment. In this line of thinking, there are many projects to apply solar heat energy in areas such as transport, residential, industrial and commercial supply, sanitation and materials recycling.

I shall present a feasible project using solar energy in large scales proposed in my doctoral thesis. As we know, solar concentrators have become a reality in the day-to-day response to sanitary and environmental preservation needs [9]. I propose a waste recycling plant as a result of years of research, unifying fundamental issues from field theory and thermodynamics in a comprehensive approach of thermal systems engineering, which is, according to Moran and colleagues, a branch of engineering concerned with how energy is utilized to get benefits in industry, transportation, the daily dealings of home, and so on [10].

Thermodynamics is a beautiful macroscopic theory, built on a few fundamental presuppositions (a fact that makes it more attractive and nice). It describes the effects of macroscopic systems formed by many microscopic entities ${ }^{1}$ that obey the basic laws of classical mechanics or quantum mechanics, as the case may be. Analyzing the generality of thermodynamics and its late claim as a solidly established physical science, we can speculate that the prevalence of mechanistic models occurred only by a matter of secular precedence of mechanics and its huge success to explain the world of the immediate things. The completeness of thermodynamics is mainly marked by its evolutionary approach of the physical systems, pointing the entropy as a fundamental variable defined in a manner somewhat abstract from a variational principle - in the process of evolution. Going from the statement that heat is energy in transit and assuming the thermodynamic equilibrium of the system as the macroscopic state for which the entropy is a maximum, it is possible to realize any physical phenomenon, insofar as the dynamics of the universe is in the end summarized by dissipation and energy exchange processes. Thus, it is also possible to historically understand the almost total lack of application of classical field theories in the context of thermodynamics, except perhaps indirectly in some specific situations where the thermal state of the system appears secondarily in the general analytical framework applied.

Present study shows a consistent application of the classical field theory in thermodynamics, focusing on the subject of recycling condensed matter, specifically in order to establish a system of solid waste treatment.

\footnotetext{
${ }^{1}$ In fact, these entities can be even infinitesimal geodesic steps, as I have verified in my model of quantum gravity, still in progress.
} 


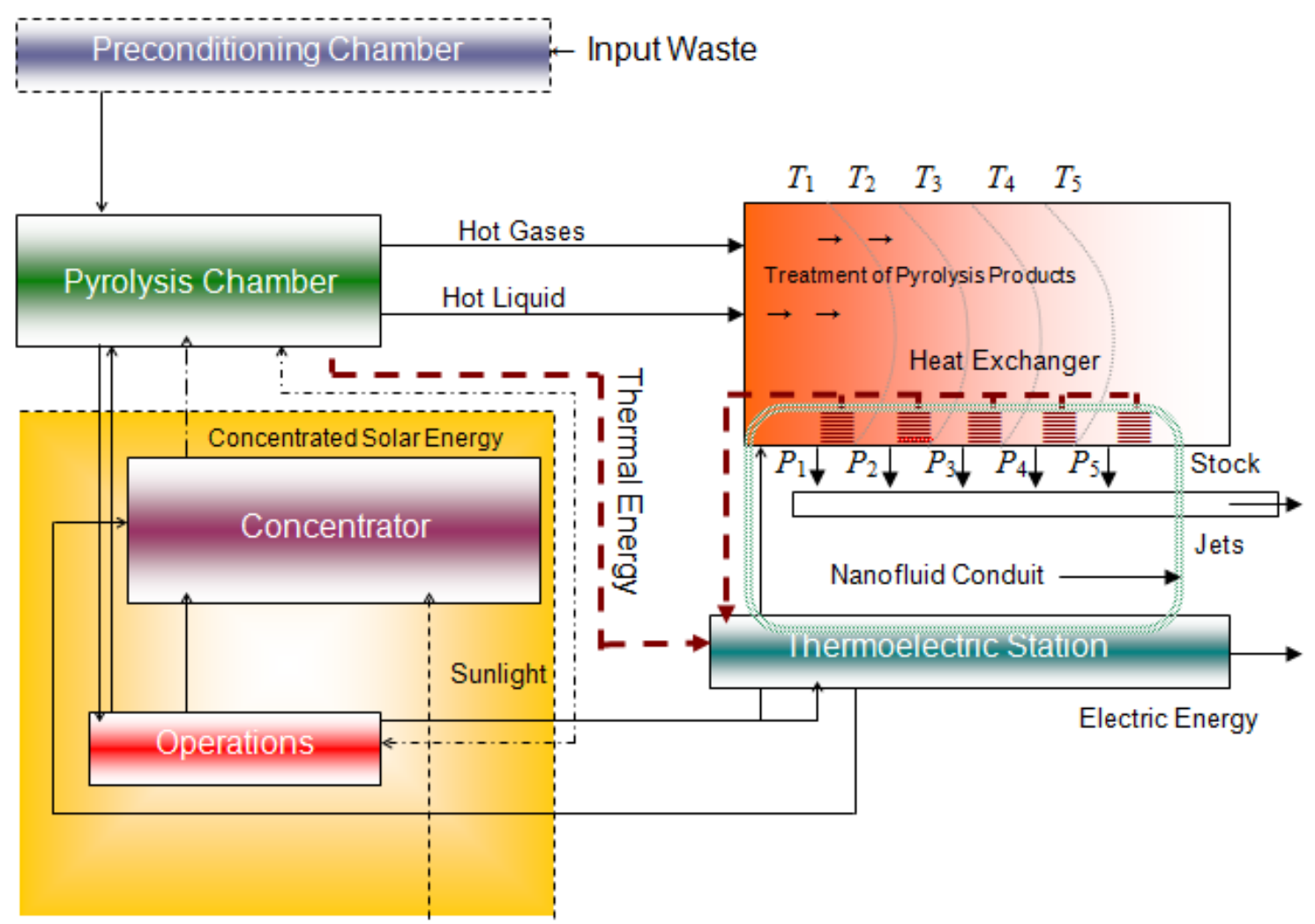

Fig. 1 The complete scheme of the proposed thermodynamic engine. Note the cycle of energy with a thermoelectric station feeding continually the plant as well as it is powered by solar energy.

Briefly, any waste fills a prototype system (Fig. 1) of two cylindrical graphite chambers in which pyrolysis and recycling processes shall take place. Internally subjected to a vacuum, first chamber (pyrolysis chamber) receives the concentrated sunlight rays from a concave array of mirrors on a quartz window placed at one of the circular bases of the chamber. At high temperatures atomic disruptions produce gases and liquids that flow to the recycling device, inside which the gradient of temperatures $T_{1}, T_{2}, T_{3}, T_{4} \ldots T_{\mathrm{n}}$ allows to a recovery of products $P_{1}, P_{2}, P_{3}, P_{4} \ldots P_{\mathrm{n}}$ from the hottest layers to the cooler. A computational control system conducts catalytic agents, whose actions enter the processes associated with temperatures to ensure the outputs of programmed materials, and the recombination of remnant atoms into inert substances in the form of usable waste. To reduce the entropy and expand the productivity of the heat generation we introduced an auxiliary piping system for the laminar flow of a nanofluid to establish a convection process of heat transfer [11, 12]. Lastly, products, final residues and usable waste are sent respectively to inventory and appropriate containment, remembering that the so called "pyrolysis ashes"- similar to the dust and blast furnace sludge - which constitute the usable waste can be used in the cement industry. All the energy needed to run the engine is solar, being the possible surplus routed to the public network.

The theoretical model developed treats the thermal energy inside the pyrolysis and recycling chambers as a complex scalar field, the so-called "caloric field" to be measured with precision and controlled at each point of its confinement for a maximum of efficiency in management of byproducts and pyrolysis process. Theoretical basis for the construction of classical fields may be found in the works of Maggiore [13] and Radovanoviç [14]. In addition, the theory and its application to the power plant forms the econophysical foundations to match operations management and environmental management in a unified operational 
level just in the sense pointed out by Kurdve et al. to include the waste management supply chain [15].

Accordingly, classical field theory, present model supposes a differential polynomial in $\xi$, the Lagrangian density $\mathcal{L}(\xi)$, given by

$$
\mathcal{L}=\left(\partial_{\mathrm{q}} \xi\right)^{*}\left(\partial^{\mathrm{q}} \xi\right)-|\xi|^{2}+2 \gamma^{2}|\xi|^{2} \ln |\xi|
$$

whose action over a certain region $\mathcal{M}$, in space and time, is

$$
\mathcal{S}(\xi)=\int_{\mathcal{M}}\left[\left(\partial_{q} \xi\right)^{*}\left(\partial^{a} \xi\right)-|\xi|^{2}+2 \gamma^{2}|\xi|^{2} \ln |\xi|\right] \mathrm{d} \mathcal{V} \mathrm{dt} .
$$

Here, from my first proposal, $\xi$ represents a scalar complex massless caloric field ${ }^{2}, d \mathcal{V}$ an infinitesimal volume of space, dt an infinitesimal time interval, and $\gamma$ a real scalar to be defined later which depends on the system's environment in question [12]. The caloric field obeys the field equation

$$
\partial_{\mathrm{q}} \partial^{\mathrm{q}} \xi+\left(1-\gamma^{2}\right) \xi-2 \gamma^{2} \xi \ln |\xi|=0
$$

being the field entropy in generalized coordinates $q$ given by

$$
\mathrm{S}=\int-2 \gamma^{2}|\xi|^{2} \ln |\xi| \mathrm{dq}
$$

Thus, field equation includes an entropy term $-2 \gamma^{2} \xi \ln |\xi|$ in the dynamics of the field and expression $\mathrm{XX}$ is just a straightforward generalization of Gibbs entropy of $\xi$. It is worth noting that for $|\xi|^{2}<1$ it follows that $2 \ln |\xi|<0$; thereby, $\mathrm{S}>0$ for every non-trivial system state. The factor

$$
\left(1-\gamma^{2}\right)
$$

in the second term of Eq. (2), the so-called "luminothermic capacity", reflects the potential power offered by the natural surroundings. Its action under the field shows how field is influenced by the external conditions. Thus, caloric field equation governs the evolution of the thermal energy field and the corresponding entropy produced.

\footnotetext{
2 Caloric field is naturally a massless field, since the temperature inside the pyrolysis chamber grows from the collision of beams of massless photons.
}

In a strictly thermodynamic theory, the fields are representations of the energy as heat, while the entropy function is a "potential". Then, we mainly deal with heat exchanges which can lead to macroscopic states where interactions between the original field and matter modify the former by the emergence of a gauge field, and so the establishment of a "massive" factor.

The fundamental idea contained in the gauge principle is that if a system remains invariant under a rigid group of continuous transformations (independent of space), then it remains invariant if the group is taken locally (depending on space). Having in mind Lagrangian formalism, we want theories where the Lagrangian density is invariant under internal symmetry transformations that depend on the point in space. If such symmetry implies a dynamic, that is, a natural description of the appropriate interactions of the theory, then there is a significant gain to the understanding of the physics running in the system under consideration. So, the application of the gauge principle consists in the introduction of new fields in the Lagrangian to remove the terms of symmetry breaking of this Lagrangian.

The usual way to present gauge theory begins with the introduction of a global continuous symmetry to the action, say an overall phase. The action does not change if we proceed the transformation

$$
\xi^{\prime} \rightarrow e^{i Q \theta} \xi
$$

The symmetry group of this transformation is the Lie group $U(1)$. On behalf of discourse economy, now we begin by the introduction of a local phase changing, say

$$
\xi^{\prime}=e^{i Q \theta(q)} \xi
$$

and its conjugate

$$
\xi^{* *}=e^{-i Q \theta(q)} \xi^{*}
$$

We rewrite our former Lagrangian

$$
\mathcal{L}_{0}=\partial_{q} \xi^{*} \partial^{q} \xi-|\xi|^{2}+\gamma^{2}|\xi|^{2} \ln |\xi|^{2} .
$$

A local transformation based on common partial differentiation gives 


$$
\begin{aligned}
& \mathcal{L}_{0}^{\prime}=\partial_{q}\left(e^{-i Q \theta(q)} \xi^{*}\right) \partial^{q}\left(e^{i Q \theta(q)} \xi\right)-|\xi|^{2}+\gamma^{2}|\xi|^{2} \ln |\xi|^{2} ; \mathcal{L}_{0}^{\prime}=\left[-i Q e^{-i Q \theta(q)} \partial_{q} \theta(q) \xi^{*}+e^{-i Q \theta(q)} \partial_{q} \xi^{*}\right] \times \\
& \times\left[i Q e^{i Q \theta(q)} \partial^{q} \theta(q) \xi+e^{i Q \theta(q)} \partial^{q} \xi\right]- \\
& -|\xi|^{2}+\gamma^{2}|\xi|^{2} \ln |\xi|^{2} \\
& \mathcal{L}_{0}^{\prime}=Q^{2} \partial_{q} \theta(q) \partial^{q} \theta(q) \xi^{*} \xi-i Q \partial_{q} \theta(q) \xi^{*} \partial^{q} \xi+i Q \partial^{q} \theta(q) \partial_{q} \xi^{*} \xi+\partial_{q} \xi^{*} \partial^{q} \xi-|\xi|^{2}+\gamma^{2}|\xi|^{2} \ln |\xi|^{2} .
\end{aligned}
$$

This operation, as we can see in the box, breaks Lagrangian invariance adding the first three terms resulting from the transformation. Consequently, we need another operator, namely the covariant derivative

$$
\mathcal{D}=\partial_{q}-i Q A_{q}\left(\text { or } \mathcal{D}=\partial_{q}+i Q A_{q}\right),
$$

with the introduction of the gauge field $A_{q}$ so that we can feature a unitary transformation as

$$
\begin{aligned}
\mathcal{L}_{0} \rightarrow \mathcal{L}_{0}^{\prime}=\left(\partial_{q}\right. & \left.+i Q A_{q}\right) e^{-i Q \theta(q)} \xi^{*}\left(\partial^{q}-i Q A^{q}\right) e^{i Q \theta(q)} \xi-e^{-i Q \theta(q)} \xi^{*} e^{i Q \theta(q)} \xi+ \\
& +\gamma^{2} e^{-i Q \theta(q)} \xi^{*} e^{i Q \theta(q)} \xi \ln \left(e^{-i Q \theta(q)} \xi^{*} e^{i Q \theta(q)} \xi\right) .
\end{aligned}
$$

The phases are canceled, leaving the short expression

$$
\begin{aligned}
& \mathcal{L}_{0} \stackrel{U(1)}{\rightarrow} \mathcal{L}_{0}^{\prime}=\left(-i Q \partial_{q} \theta \xi^{*}+\partial_{q} \xi^{*}+i Q A_{q} \xi^{*}\right)\left(i Q \partial^{q} \theta \xi+\partial^{q} \xi-i Q A^{q} \xi\right)- \\
& -\xi^{*} \xi+\gamma^{2} \xi^{*} \xi \ln \left(\xi^{*} \xi\right) \text {. }
\end{aligned}
$$

Making up multiplications term-to-term we obtain

$$
\begin{gathered}
\mathcal{L}_{0} \stackrel{U(1)}{\rightarrow} \mathcal{L}_{0}^{\prime}= \\
\frac{Q^{2} \partial_{q} \theta \partial^{q} \theta \xi^{*} \xi-i Q \partial_{q} \theta \xi^{*} \partial^{q} \xi-Q^{2} A^{q} \partial_{q} \theta \xi^{*} \xi+}{+i Q \partial^{q} \theta \partial_{q} \xi^{*} \xi+\partial_{q} \xi^{*} \partial^{q} \xi-i Q A^{q} \partial_{q} \xi^{*} \xi-} \\
-Q^{2} A_{q} \partial^{q} \theta \xi^{*} \xi+i Q A_{q} \xi^{*} \partial^{q} \xi+Q^{2} A_{q} A^{q} \xi^{*} \xi-\xi^{*} \xi+\gamma^{2} \xi^{*} \xi \ln \left(\xi^{*} \xi\right),
\end{gathered}
$$

where I kept boxed terms that shall be canceled. However, based on the above development, this cancellation comes from the potential introduction in the expression

$$
\begin{aligned}
& \mathcal{L}_{\text {Cauge }}=-Q^{2} A^{q} \partial_{q} \theta \xi^{*} \xi-i Q A^{q} \partial_{q} \xi^{*} \xi- \\
& -Q^{2} A_{q} \partial^{q} \theta \xi^{*} \xi+i Q A_{q} \xi^{*} \partial^{q} \xi+Q^{2} A_{q} A^{q} \xi^{*} \xi,
\end{aligned}
$$

which we called "gauge Lagrangian”. Then, we have

$$
A_{q}^{\prime}=A_{q}+\partial_{q} \theta
$$

from which 


$$
\begin{gathered}
\mathcal{L}_{\text {Cauge }}^{\prime}=-Q^{2}\left(A^{q}+\partial^{q} \theta\right) \partial_{q} \theta \xi^{*} \xi-i Q\left(A^{q}+\partial^{q} \theta\right) \partial_{q} \xi^{*} \xi- \\
-Q^{2}\left(A_{q}+\partial_{q} \theta\right) \partial^{q} \theta \xi^{*} \xi+ \\
+i Q\left(A_{q}+\partial_{q} \theta\right) \xi^{*} \partial^{q} \xi+Q^{2}\left(A_{q}+\partial_{q} \theta\right)\left(A^{q}+\partial^{q} \theta\right) \xi^{*} \xi .
\end{gathered}
$$

The reader must note that the gauge field $A_{q}$ does not transform by covariant mode. The way it transforms comes from the requirement

$$
\begin{gathered}
\left(\mathcal{D}_{q} \xi\right)^{\prime}=\left(\partial_{q}-i Q A_{q}^{\prime}\right) \xi ; \\
\left(\mathcal{D}_{q} \xi\right)^{\prime}=\left(\partial_{q}-i Q A_{q}^{\prime}\right) e^{i Q \theta(q)} \xi ; \\
\left(\mathcal{D}_{q} \xi\right)^{\prime}=e^{i Q \theta(q)}\left(\partial_{q} \xi+i Q \partial_{q} \theta(q) \xi-i Q A_{q}^{\prime} \xi\right) ; \\
\left(\mathcal{D}_{q} \xi\right)^{\prime}=e^{i Q \theta(q)}\left[\partial_{q}+i Q \partial_{q} \theta(q)-i Q\left(A_{q}+\partial_{q} \theta(q)\right)\right] \xi ; \\
\left(\mathcal{D}_{q} \xi\right)^{\prime}=e^{i Q \theta(q)}\left[\partial_{q}+i Q \partial_{q} \theta(q)-i Q A_{q}-i Q \partial_{q} \theta(q)\right] \xi ; \\
\left(\mathcal{D}_{q} \xi\right)^{\prime}=e^{i Q \theta(q)}\left(\partial_{q}-i Q A_{q}\right) \xi .
\end{gathered}
$$

Usually it is assumed that $A_{q}$ describes some new and independent degrees of freedom of the system. By applying the change, it follows that

$$
\begin{aligned}
\mathcal{L}_{\text {Gauge }}^{\prime} & =\left[\left[-Q^{2} A^{q} \partial_{q} \theta \xi^{*} \xi-Q^{2} \partial^{q} \theta \partial_{q} \theta \xi^{*} \xi\right]\right]- \\
& -i Q A^{q} \partial_{q} \xi^{*} \xi-i Q \partial^{q} \theta \partial_{q} \xi^{*} \xi+ \\
+ & {\left[\left[-Q^{2} A_{q} \partial^{q} \theta \xi^{*} \xi\right]\right]-Q^{2} \partial_{q} \theta \partial^{q} \theta \xi^{*} \xi+} \\
+ & +i Q A_{q} \xi^{*} \partial^{q} \xi+i Q \partial_{q} \theta \xi^{*} \partial^{q} \xi+ \\
+Q^{2} A_{q} A^{q} \xi^{*} \xi & +\left[\left[Q^{2} A_{q} \partial^{q} \theta \xi^{*} \xi+Q^{2} \partial_{q} \theta A^{q} \xi^{*} \xi+Q^{2} \partial_{q} \theta \partial^{q} \theta \xi^{*} \xi\right]\right] .
\end{aligned}
$$

Since the theory is Abelian, the order of the factors in the multiplication does not matter. Terms in double brackets are canceled naturally, while boxed terms cancel the terms of symmetry breaking of the former Lagrangian. So,

$$
\begin{aligned}
\mathcal{L}_{0}^{\prime} & +\mathcal{L}_{\text {gauge }}^{\prime}=\partial_{q} \xi^{*} \partial^{q} \xi-|\xi|^{2}+\gamma^{2}|\xi|^{2} \ln |\xi|^{2}+ \\
& +Q^{2} A_{q} A^{q} \xi^{*} \xi+i Q\left(A_{q} \xi^{*} \partial^{q} \xi-A^{q} \partial_{q} \xi^{*} \xi\right)
\end{aligned}
$$

or

$$
\begin{aligned}
& \mathcal{L}_{0}^{\prime}+\mathcal{L}_{\text {gauge }}^{\prime}=\partial_{q} \xi^{*} \partial^{q} \xi-|\xi|^{2}+\gamma^{2}|\xi|^{2} \operatorname{In}|\xi|^{2}+ \\
& +Q^{2} A_{q} A^{q} \xi^{*} \xi+i Q\left\{A_{q} \partial^{q}, A^{q} \partial_{q}\right\}_{\xi^{*}, \xi}
\end{aligned}
$$


Additional terms that express the interactions between fields carry the generator of the symmetry group of the theory. Since the field $A_{q}$ is added to our Lagrangian as a tool to assert gauge invariance of the caloric field kinetic term, we must recognize the need to add a kinetic term for the gauge field itself. We introduce a field strength tensor, built from the commutator of covariant derivatives

$$
\begin{gathered}
{\left[D_{p}, D_{q}\right]=\left[\left(\partial_{q}-i Q A_{q}\right)\left(\partial_{p}-i Q A_{p}\right)\right]-\left[\left(\partial_{p}-i Q A_{p}\right)\left(\partial_{q}-i Q A_{q}\right)\right]} \\
{\left[D_{p}, D_{q}\right]=\left[\partial_{q} \partial_{p}-i Q \partial_{q} A_{p}-i Q A_{q} \partial_{p}-Q^{2} A_{q} A_{p}\right]-} \\
-\left[\partial_{p} \partial_{q}-i Q \partial_{p} A_{q}-i Q A_{p} \partial_{q}-Q^{2} A_{p} A_{q}\right] \\
{\left[D_{p}, D_{q}\right]=\left[-i Q \partial_{q} A_{p}-i Q A_{q} \partial_{p}\right]-\left[-i Q \partial_{p} A_{q}-i Q A_{p} \partial_{q}\right]} \\
{\left[D_{p}, D_{q}\right]=i Q\left(\partial_{p} A_{q}-\partial_{q} A_{p}\right)=i Q \mathcal{F}_{p q} .}
\end{gathered}
$$

The new kinetic term must also preserve Lorentz invariance, so that it assumes the form

$$
\mathfrak{F}=\mathcal{F}_{p q} \mathcal{F}^{p q} .
$$

Thus, we have a new code for the Lagrangian with embedded transformation of the gauge field, which is

$$
\mathfrak{L}_{0}^{\prime}=\mathcal{D}_{q} \xi^{*} \mathcal{D}^{q} \xi-|\xi|^{2}+\gamma^{2}|\xi|^{2} \ln |\xi|^{2}-\mathfrak{F}
$$

Given that the kinetic terms of the classical fields involved do not originate from a mechanical model, there is in principle no reason to assume fractional constants in these terms. Now, the question to ask is: what is the need for a gauge approach to this classic situation in a "so familiar" terrain like thermodynamics? The answer depends on a correct physical intuition, as on the almost inexhaustible capacity for representation of the physical-mathematical formalism. We have already shown that an unconventional approach to thermal energy is possible. If the massless caloric field, as presented above, was simply generated in a vacuum, nothing new would take place. However, when interacting with the mass of waste, the field generates mass for itself, since the thermo-physical and chemical reactions triggered generate heat providing thermal feedback to the former caloric field, plus a small amount of volatile mass assimilated by the field. This mass is then represented by the constant of minimum coupling with the gauge field, called "Q" (the symmetry group generator), something like a "caloric charge" or better yet "minimal thermal mass factor of dynamic interaction”. We note that this corresponding generator does not respect the former vacuum of the chamber.

Thereby, the introduction of the gauge field discovers a new physics, free from derivatives on this field, namely, the interactions that are triggered by the action of the field on the waste which could not exist before introducing the material into the pyrolysis chamber. Indeed, gauge field $A_{q}$ mediates a "strain"3 between the fields (and their derivatives) with coupling Q. The symmetry varies point to point, since the processes are subject to a gradient of temperatures and a random volatilization of matter (the phase of the field function can be chosen arbitrarily at each space-time point). This information should be part of the stochastic processing algorithm to be initialized in "Operations" (Fig. 1) in order to accurately calculate the amount of

\footnotetext{
3 We can say that a local symmetry generates a "strain" coupled to the "caloric charge". In other words, inside the chamber, if we gauge caloric energy and the minimal thermal mass of dynamic interaction, we shall get forces (internal pressures that can be attributed to the shock of the pyrolytic plasma molecules against the walls of the chamber, thereby being transmitted impulse to the walls) for which the sources are the energy and momentum of the molecules.
} 
non-recycled material, and the mass percentage assimilated into the field. Lastly, with the recycling of all materials from pyrolysis, the remaining pyrolytic ashes feature a completely inert environment within the chambers. In this situation, the Lagrangian density interactions sector must be annulled.

\section{Narrowing the Ideas of Entropy Reduction and Cleaner Production}

At large scale of time, entropy is a magnitude that increases for dynamic systems; it is founded on the counting of disordered and ordered situations, which leads to the conclusion that it is very improbable the change of a system by spontaneous behavior from a disordered to an ordered state. Entropy is so related to the general notion that dynamic systems evolve towards more probable states. Since closed dynamic systems are abstractions not feasible in our daily life, we may neglect theoretical considerations about such systems, concentrating attention on realistic situations in which energy interchanging is an obvious fact.

The generation of entropy is associated with a certain amount of hidden information that would be required for the system to evolve in a specific known path, that is, in an organized evolutionary track. It is exactly this information that will be processed and controlled by computerized operating module coupled to the processing chamber (Fig. 1).

For an open system, the relation between information entropy and system entropy generation is given by

$$
\mathrm{S}_{\mathrm{G}}=\mathrm{S}=-\mathrm{k} \int \mathrm{P}_{\mathrm{p}}(\mathrm{x}, \mathrm{t}) \ln \left(\mathrm{P}_{\mathrm{p}}(\mathrm{x}, \mathrm{t})\right) \mathrm{dx}
$$

in which $P_{\hat{p}}(x, t)$ is the probability distribution of a path $\hat{p}$, and $k$ is a proportionality constant. More probable states are states of increased disorder that point to system's degradation. For instance, the supposed future Universe with no light, no matter or energy, shall be in maximal state of degradation. Whereas the entropy of a dynamical system always tends to grow in the direction of time, it is reasonable to assume that, at best, control actions only decelerate the advancement of entropy. Thus, it is appropriate to talk about a variation in entropy. Looking at presented engine, the total variation in the generation of entropy may be written as

$$
\delta \mathrm{S}_{\mathrm{tot}}=\delta \mathrm{S}_{\mathrm{int}} \pm \delta \mathrm{S}_{\mathrm{ext}},
$$

where $\delta \mathrm{S}_{\text {int }}$ is the internal entropy variation, and $\delta S_{\text {ext }}$ is the variation of entropy due to the exchanging process between the system and the external world. For a high disordered system, $\delta \mathrm{S}_{\text {ext }}>0$ is minimal because there are almost no organized outputs to be registered; only inputs are generated at random manner. So,

$$
\delta \mathrm{S}_{\mathrm{tot}} \simeq \delta \mathrm{S}_{\mathrm{int}} .
$$

If random inputs are continuously generated, $\delta \mathrm{S}_{\text {int }}$ increases, so does $\delta \mathrm{S}_{\text {ext }}$. To minimize total entropy variation, we need $\delta \mathrm{S}_{\text {ext }}>0$, so that we may write

$$
\delta \mathrm{S}_{\mathrm{tot}}=\delta \mathrm{S}_{\mathrm{int}}-\delta \mathrm{S}_{\mathrm{ext}} .
$$

This means that ordered outputs are returned to the environment and general entropy grows more slowly. The immediate interpretation of this fact is that production is now cleaner since inputs are organized to attain an objective final result. The random aspect of the inputs is now compensated by rational processing of waste entries.

Lastly, the application of the gauge principle as set forth above establishes in fact an additional "control principle" of the thermo-chemical and physical processes that occur in the plant, offering theoretical elements to be included in the stochastic algorithms of computational control in the sector "Operations".

\section{Conclusions}

The destination of urban waste is a great problem over the entire world. The modern SWM needs to seriously consider the use of renewable energy sources to disposal waste so that we can talk about sustainable 
environments. Initial investments are certainly high, but the return from medium to long term is proportionally much larger. The main contribution of this work was a realistic proposal for the use of solar energy to recycling and disposal waste with very low environmental impacts. The proposal was centered on the fact that modern high-temperature incinerators are not solutions, neither from the viewpoint of local security, nor from the viewpoint of environmental security. Presented model is an environmentally responsible proposal to employ the energy source more evident that we have — the sun — to empower a device for treating waste by disaggregation of materials at the atomic level. This process also allows the recovery of recyclable materials, as well as the generation of by-products usable in other contexts of production. The application of the gauge principle in classical field theory was the core of the theoretical background of the proposal. As we have seen, this principle is so powerful that we can appreciate it in thermodynamics applied to engineering systems. It is expected that the model gives rise to power plants to be implemented at the most problematic metropolitan locations in terms of generation and disposal of urban waste.

\section{Acknowledgements}

The author would like to thank Professor Dr. José Abdalla Helayël-Neto, at Brazilian Center for Physics Research, for the support and important observations. The author also thanks Professor Dr. Antonio Fernandes da Fonseca Teixeira, at Brazilian Center for Physics Research, by the time generously dedicated to some important debates.

\section{References}

[1] Abah, S., and Ohimain, E. 2011. "Healthcare Waste
Management in Nigeria-A Case Study.” Journal of Public Health \& Epidemiology 3: 99-110.

[2] Abd El-Salam, M. 2010. "Hospital Waste Management in El-Beheira Governorate-Egypt.” Journal of Environmental Management 91: 618-29.

[3] Ananth, P., Prashanthini, V., and Visvanathan, C. 2010. "Healthcare Waste Management in Asia." Waste Management 30: 154-61.

[4] Mato, R., and Kaseva, M. 1999. "Critical Review of Industrial and Medical Waste Practices in Dar es Salaam City.” Resources, Conservation and Recycling 25: 271-87.

[5] Rahman, H. 2013. "Incinerator in Malaysia-Really Needs?” International Journal of Chemical, Environmental \& Biological Sciences 1 (4): 678-81.

[6] Reddy, P. 2011. Municipal Solid Waste Management. Boca Raton: CRC Press, 449p.

[7] ICRC-International Committee of the Red Cross. 2011. Medical Waste Management. Geneva, Switzerland.

[8] Tsakona, M., Anagnostopoulou, E., and Gidarakos, E. 2007. "Hospital Waste Management and Toxicity Evaluation: A Case Study." Waste Management 27: 912-20.

[9] Kashyap, A., Kesari, J., Rai, A., and Singh, H. 2014. "Study of Designing a Solar Concentrator for Crematorium Using Solar Energy.” International Journal of Advance Research and Innovation 2 (3): 564-7.

[10] Moran, M., Shapiro, H., Munson, B., and DeWitt, D. 2003. Introduction to Thermal Systems Engineering. New York: John Wiley \& Sons, Inc.

[11] Eastman, J., Phillpot, S., Choi, S., and Keblinski, P. 2004. "Thermal Transport in Nanofluids." Annual Review of Materials Research 34: 219-46.

[12] Serpa, N. 2014. “Sur L’Entropie Contrôlée des Systèmes.” Ph.D. Thesis, L’Université Libre des Sciences de L'Homme de Paris, 127p.

[13] Maggiore, M. 2005. A Modern Introduction to Quantum Field Theory. New York: Oxford Master Series in Physics, Oxford University Press.

[14] Radovanoviç, V. 2008. Problem Book in Quantum Field Theory. Berlim: Springer-Verlag.

[15] Kurdve, M., Shahbazi, S., Wendin, M., Bengtsson, C., and Wiktorsson, M., 2015. "Waste Flow Mapping to Improve Sustainability of Waste Management-A Case Study Approach." Journal of Cleaner Production 98: 304-15. 